\title{
Epidemiological and Clinical Characteristics of Alveolar Echinococcosis: An Emerging Infectious Disease in Alberta, Canada
}

\author{
Stan Houston, ${ }^{1 \star}$ Sara Belga, ${ }^{1,2}$ Klaus Buttenschoen, ${ }^{1}$ Ryan Cooper, ${ }^{1}$ Safwat Girgis, ${ }^{1}$ Bruno Gottstein, ${ }^{3}$ Gavin Low, ${ }^{1}$ \\ Alessandro Massolo, ${ }^{4}$ Clayton MacDonald, ${ }^{1}$ Norbert Müller, ${ }^{3}$ Jutta Preiksaitis, ${ }^{1}$ Philippe Sarlieve, ${ }^{1}$ Stephen Vaughan, ${ }^{5}$ and \\ Kinga Kowalewska-Grochowska ${ }^{1}$ \\ ${ }^{1}$ University of Alberta, Edmonton, Canada; ${ }^{2}$ University of British Columbia, Vancouver, Canada; ${ }^{3}$ University of Bern, Bern, Switzerland; ${ }^{4}$ University \\ of Pisa, Pisa, Italy; ${ }^{5}$ University of Calgary, Calgary, Canada
}

\begin{abstract}
Human alveolar echinococcosis (AE) is a zoonotic cestode infection which is usually fatal in the absence of treatment. Treatment involves major surgery or indefinite antiparasitic therapy. The incidence is rising in Europe and Asia, with an increased risk observed in immunocompromised individuals. Previously, AE acquisition in North America was extremely rare, except for one remote Alaskan Island. Recent studies have demonstrated a new European-like strain of Echinococcus multilocularis $(E m)$ in wildlife and in human AE in western Canada. We report the experience of all $A E$ patients diagnosed in Alberta. Each was diagnosed by histopathology, serology, and PCR-confirmed by a reference laboratory. Seventeen cases of human AE, aged 19-78 years, nine females, were diagnosed between 2013 and 2020 : all definitely or probably acquired in Alberta. Six lived in urban areas, and 14 had kept dogs. In eight, the lesions were found incidentally on abdominal imaging performed for other indications. Six were immunocompromised to varying degrees. Six were first diagnosed at surgery. All have been recommended benzimidazole therapy. One died of surgical complications. Clinicians should be aware of this diagnostic possibility in patients presenting with focal nonmalignant hepatic mass lesions. Greater urbanization of coyotes, the predominant definitive host of Em in Alberta, and growing numbers of immune suppressed individuals in the human population may lead to increasing recognition of AE in North America.
\end{abstract}

\section{INTRODUCTION}

Echinococcus multilocularis $(E m)$ is the etiological agent of human alveolar echinococcosis (AE), a globally important cestode parasite ${ }^{1,2}$ with more than 18,000 cases/year and an estimated burden of 660,000 disability-adjusted life years. ${ }^{3}$ The first human cases were reported from southern Germany in 1852, and a parasite etiology was recognized by Rudolf Virchow in $1855 .^{4}$

The 2-3-mm adult worms live in the small intestine of the definitive hosts-commonly canids (fox, coyote, wolf, or domestic dog), less frequently cats, which are not thought to contribute significantly to transmission, and eggs are shed in feces. Once ingested by the intermediate host, usually a small rodent, the eggs hatch and larvae migrate to the liver where they develop into a multivesicular, infiltrating metacestode stage, subsequently producing infective protoscolices. These in turn develop into adult tapeworms when the intermediate host is preyed upon by a definitive host. ${ }^{5}$ Domestic dogs occasionally act as aberrant intermediate hosts, developing $\mathrm{AE}$ with progressive liver lesions. ${ }^{6}$ Humans are infected on accidental ingestion of eggs from contaminated food or soil, or from close contact with canids which have fur contaminated by parasite eggs associated with intestinal infection or the "scent rolling" behavior. ${ }^{7}$

The incubation period of $A E$ in humans is thought to be up to 15 years. ${ }^{8}$ The asexual replication of metacestodes produces a slowly infiltrating tumor-like mass which involves the liver in almost all cases, but with the potential for local invasion and distant metastatic spread. Recent reports from Europe indicate that immunocompromised patients are at increased risk for $A E$ and may manifest a shorter incubation period and atypical presentations. ${ }^{9,10}$ The natural history, radiographic

* Address correspondence to Stan Houston, University of Alberta, 1124 Clinical Sciences Bldg., 1135083 Ave., Edmonton T6G 2G3, Canada. E-mail: shouston@ualberta.ca appearance, diagnosis, and management of $A E$ are quite distinct from those of cystic echinococcosis (hydatid disease) caused by members of the Echinococcus granulosus complex.

Advanced $A E$ may present with malaise, fatigue, weight loss, right upper quadrant discomfort, and/or jaundice, but asymptomatic early stages of the disease are often diagnosed following an incidental finding on abdominal imaging for an unrelated indication. Diagnosis requires a high level of suspicion in a patient with abnormal liver imaging followed by confirmation by histopathology, serology, and PCR. ${ }^{11}$

Mortality is high without treatment, but outcomes have improved markedly with current medical and surgical management. ${ }^{11}$ Complete surgical excision is the treatment of choice when technically feasible. Non-curative "debulking" surgery does not appear to be beneficial. ${ }^{12}$ The use of benzimidazole agents is indicated in all patients; indefinite therapy is necessary when definitive surgery is not possible because these agents are only parasitostatic. ${ }^{11}$ Albendazole is generally recommended because its systemic absorption is better than that of mebendazole. No other agent has been shown to be effective in human $A E .{ }^{13}$ Liver transplantation has been used in a small number of patients. ${ }^{14}$

\section{EMERGENCE OF A NEW STRAIN OF ENZOOTIC ECHINOCOCCUS MULTILOCULARIS IN CANADA}

The endemic North American sub-strains of Em, N1 (tundra zone), and N2 (central regions), which are genetically distinct from the European or Asian strains, have long been recognized in animals in North America. ${ }^{15,16}$ However, only two cases of autochthonous human disease had previously been confirmed in North America outside of St. Lawrence Island, Alaska-one in Manitoba, Canada, in $1928^{17}$ and one in Minnesota, USA, in 1977. ${ }^{18,19}$ The latter case was retrospectively shown by molecular typing to have been caused by the N2 strain of the parasite. ${ }^{18}$ The endemic focus in St. Lawrence 
Island, Alaska, appears to be associated with an Asian strain of the parasite. ${ }^{20}$ Recently, a human case with genetic characteristics of the European strain was diagnosed in Vermont. ${ }^{21}$

The assumption that the endemic North American strain had low virulence for humans is supported by the consistent paucity of human $A E$ cases on the continent, by animal studies, ${ }^{22}$ and by the absence of serologic evidence of infection in trappers at high risk of exposure in North America. ${ }^{23}$

Since 2012, the presence of a novel strain of Em has been documented in definitive hosts, primarily coyotes, and rodent intermediate hosts in western Canada. This strain has been shown to be genetically very close to the European strain but distant from the previously endemic N2 strain. ${ }^{15,24-27}$

In the first seven cases of human AE (Table 1; patients 1-7), molecular typing was available from tissue samples of five. All five showed the presence of a European-like Em strain. In four of these from whom adequate material was available, a single nucleotide polymorphism (SNP) in position 235 of the cob gene, which has been shown to be unique to North American specimens and predominant in local wildlife, was found. ${ }^{24}$

We describe the epidemiological and clinical characteristics of human AE cases diagnosed over 7 years in Alberta, Canada, including the clinical and epidemiologic characteristics of the seven cases whose molecular epidemiology has been reported. ${ }^{28}$

\section{METHODS}

Diagnosis was initiated in every case following abnormal hepatic imaging, whether an incidental finding in the course of investigation for other conditions or precipitated by symptoms likely caused by AE. All cases were confirmed according to the criteria established by the WHO Informal Working Group on Echinococcosis, and by imaging, tissue histopathology, serology, and PCR. ${ }^{11}$ Serology and PCR were performed at the Institute of Parasitology, Vetsuisse Faculty, the University of Bern, Switzerland. Serologic testing used in-house Em2 ELISA $^{29}$ and Em18 ELISA, ${ }^{30}$ which were confirmed by inhouse Western blot. ${ }^{31}$ Multiplex PCR was carried out on gDNA isolated from formalin-preserved core needle biopsies ${ }^{32}$ or from gDNA directly isolated from resected tissue according to Trachsel et al. ${ }^{33}$ Each patient was interviewed regarding the place of residence, detailed travel history, dog ownership, and potential environmental exposure.

\section{RESULTS}

Seventeen human AE cases were diagnosed in Alberta, Canada, between 2013 and 2020 (Table 1). In terms of exposure risks, 15 were long-term dog owners, and 12 had lived predominantly in rural areas (Figure 1). One patient (patient 4) had performed autopsies on wolves, and three patients (patients 11, 12, and 17) were farmers who described frequent exposure to coyote feces and/or carcasses. Nine had never travelled to any part of Europe or Asia known to be endemic for $\mathrm{AE}$, and in several of those with a European travel history, the duration was very short (e.g., 2 weeks in Paris) or the timing of exposure (e.g., 44 years before diagnosis) was extremely unlikely to be consistent with Europe having been the site of $E m$ infection. There were no clusters or linked cases.

Six patients were immunosuppressed to varying degrees: one renal transplant recipient (patient 1), one on methotrexate for psoriatic arthritis (patient 4), one on corticosteroids for inflammatory bowel disease (patient 5), one on methotrexate and prednisone for inflammatory arthritis (patient 8), one who had undergone chemotherapy and radiation for metastatic colon cancer (patient 11), and one who had a stem-cell transplant and chemotherapy for myeloma (patient 14).

TABLE 1

Demographic characteristics, epidemiologic risk factors, clinical features, diagnostic results, disease classification, and treatment interventions of 17 cases of alveolar echinococcosis in Alberta, Canada

\begin{tabular}{|c|c|c|c|c|c|c|c|c|c|c|c|c|}
\hline $\mathrm{Pt}$ & $\begin{array}{c}\text { Age (years)/year of } \\
\text { diagnosis }\end{array}$ & Gender & $\begin{array}{l}\text { Travel: Europe } \\
\text { or Asia }\end{array}$ & $\begin{array}{l}\text { Dog } \\
\text { owner }\end{array}$ & $\begin{array}{l}\text { Rural// } \\
\text { urban }\end{array}$ & Immunocompromised & $\begin{array}{l}\text { Incidental } \\
\text { finding }\end{array}$ & $\begin{array}{l}\text { \# Of } \\
\text { lesions }\end{array}$ & $\begin{array}{c}\text { IWGE-WHO stage } \\
(10,40)\end{array}$ & Serology & PCR & Therapy \\
\hline 1 & $49 / 2013$ & Female & Yes & Yes & Rural & Yes & Yes & 7 & P3N0MO IIIa & POS & POS & Albendazole \\
\hline 2 & $47 / 2016$ & Female & Yes & Yes & Rural & No & No & 4 & P4N1M0 IV & POS & POS & Albendazole \\
\hline 3 & $78 / 2016$ & Male & No & Yes & Urban & No & $?$ & 1 & P1MON0I & POS & POS & $\begin{array}{l}\text { Surgery and } \\
\text { albendazole }\end{array}$ \\
\hline 4 & $52 / 2016$ & Female & No & Yes & Rural & Yes & No & 2 & P1N0M0 I & IND & POS & $\begin{array}{l}\text { Surgery and } \\
\text { albendazole }\end{array}$ \\
\hline 5 & $19 / 2017$ & Male & Yes & Yes & Urban & Yes & Yes & 3 & P1N0M0 I & IND & POS & $\begin{array}{l}\text { Surgery none } \\
\text { (refused) }\end{array}$ \\
\hline 6 & $55 / 2018$ & Male & Yes & Yes & Urban & No & Yes & 5 & P1N0M0 I & POS & POS & Albendazole \\
\hline 7 & $65 / 2018$ & Female & Yes & Yes & Rural & No & No & 2 & P1N1M0 IIIb & POS & POS & $\begin{array}{l}\text { Surgery and } \\
\text { mebendazole }\end{array}$ \\
\hline 8 & $52 / 2018$ & Female & Yes & Yes & Rural & Yes & No & 2 & P1N1M0 IIIb & POS & POS & $\begin{array}{l}\text { Surgery and } \\
\text { albendazole }\end{array}$ \\
\hline 9 & $57 / 2018$ & Male & No & Yes & Rural & No & Yes & 1 & P1N0MO I & POS & POS & $\begin{array}{l}\text { Surgery and } \\
\text { albendazole }\end{array}$ \\
\hline 10 & $75 / 2018$ & Female & Yes & No & Urban & No & Yes & 3 & P3NOMO I & NEG & POS & Albendazole \\
\hline 11 & $56 / 2018$ & Male & No & Yes & Rural & Yes & Yes & 3 & P1N0MO I & NEG & POS & $\begin{array}{l}\text { Surgery and } \\
\text { albendazole }\end{array}$ \\
\hline 12 & $68 / 2018$ & Male & No & Yes & Rural & No & No & 2 & P4N1M0 IV & POS & NEG & Albendazole \\
\hline 13 & $60 / 2019$ & Female & Yes & No & Urban & No & Yes & 1 & P4N1MXIV & POS & POS & Albendazole \\
\hline 14 & $72 / 2019$ & Male & No & Yes & Rural & Yes & No & 4 & P2N0M1 IV & POS & POS & Albendazole \\
\hline 15 & $36 / 2019$ & Female & No & Yes & Rural & No & No & 1 & P4N1M1.IV & POS & POS & $\begin{array}{l}\text { Surgery and } \\
\text { albendazole }\end{array}$ \\
\hline 16 & $66 / 2020$ & Female & No & Yes & Rural & No & No & 5 & P3N0MO IIla & POS & POS & Albendazole \\
\hline 17 & $62 / 2020$ & Male & No & Yes & Rural & No & Yes & 1 & P3NOMOI & POS & POS & Surgery; died \\
\hline
\end{tabular}

NEG = negative; POS = positive; IWGE-WHO = World Health Organization Informal Working Group on Echinococcosis. 


\section{CASE MAP: ALBERTA CANADA}

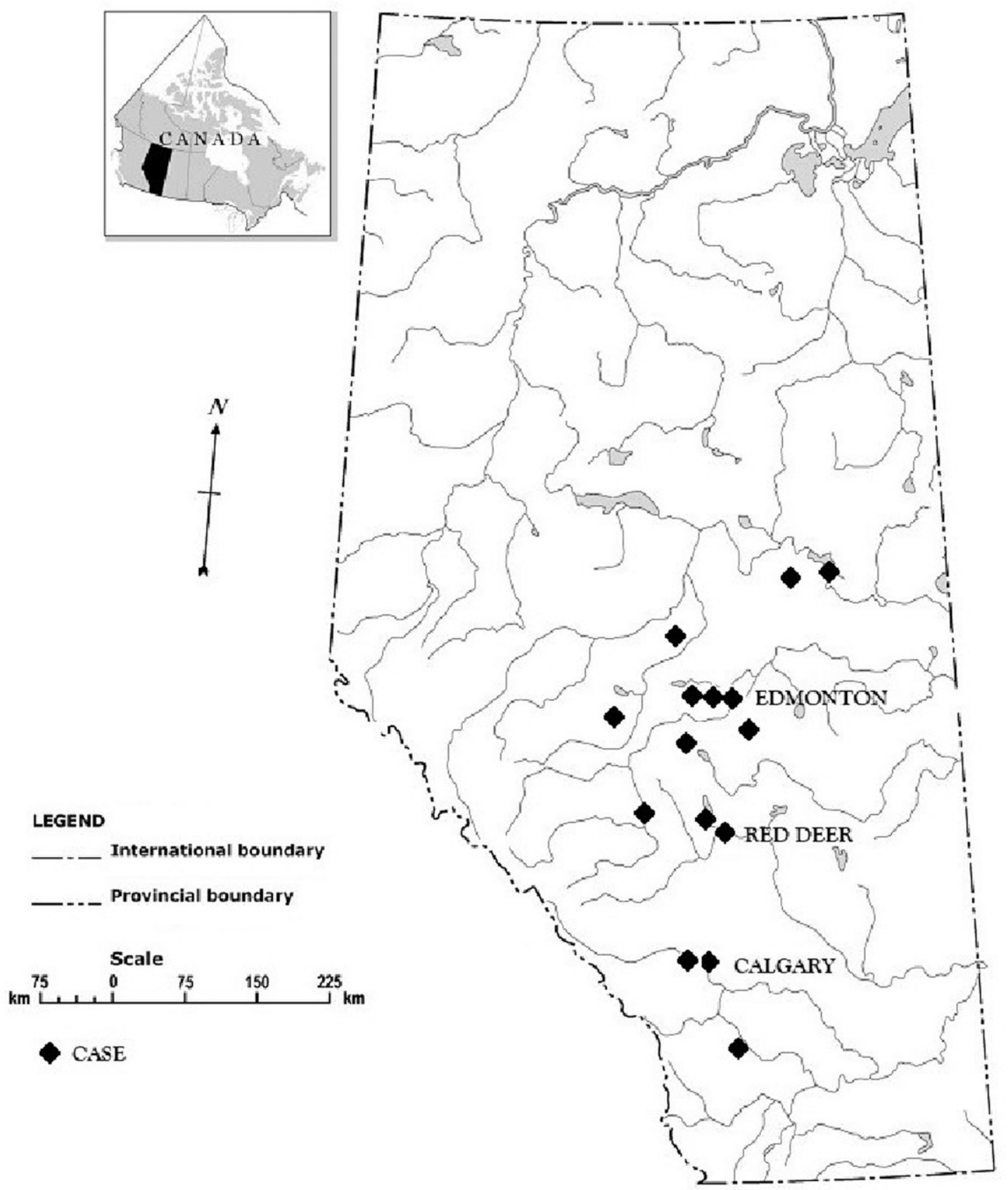

\section{MONTANA, USA}

FIGURE 1. Map of case distribution of alveolar echinococcus cases within the province of Alberta. Insert: location of the province of Alberta within Canada. Symbols: cases are represented by diamonds, and major urban centers are denoted by name. 

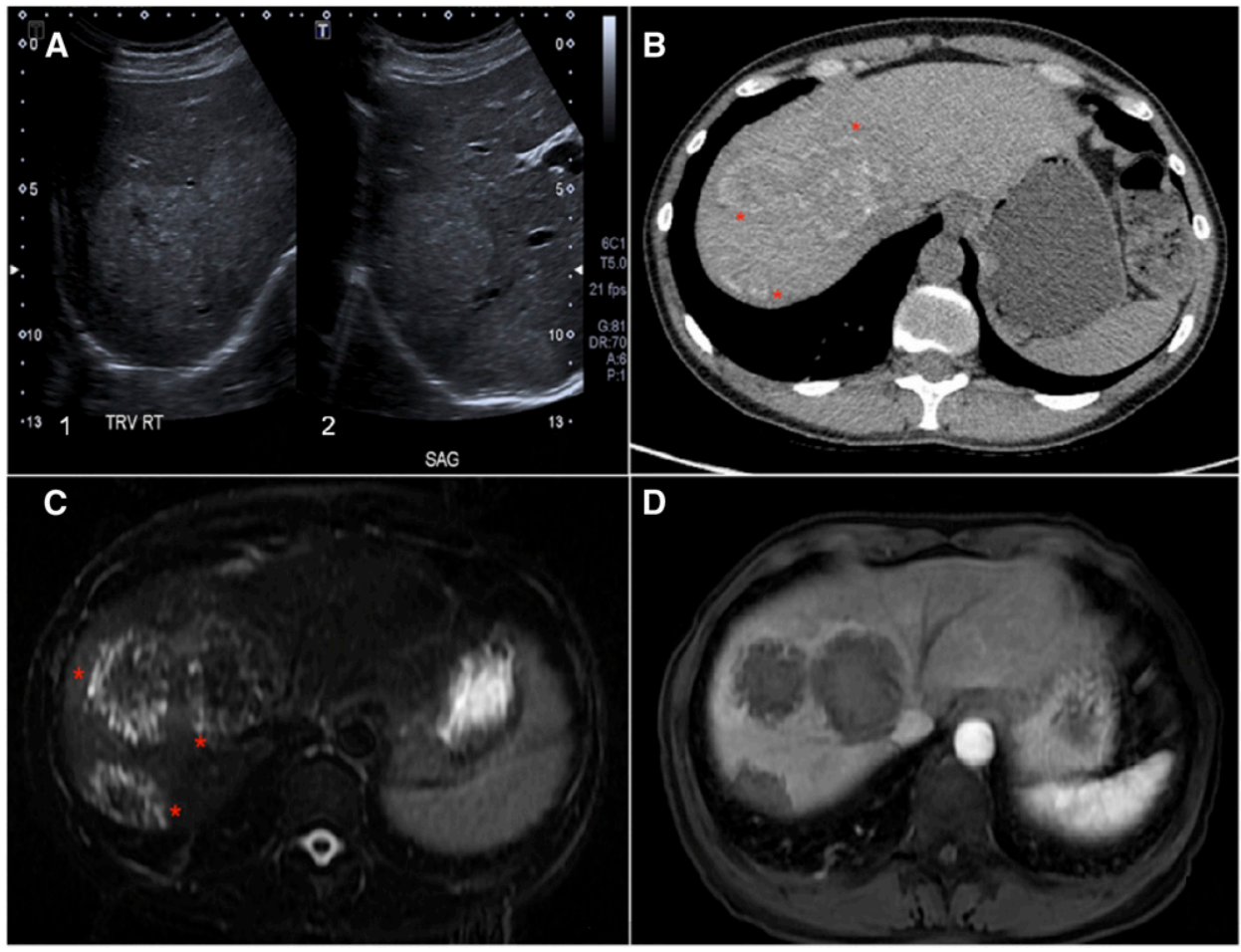

FIGURE 2. A 49-year-old female with biopsy-proven Echinococcus multilocularis (Em) (patient 2). A dual screen capture from an abdominal sonogram on the same patient. (A-1) Transverse and (A-2) sagittal images of the right hepatic lobe demonstrate that the lesions are heterogeneously echogenic compared with the background liver. There are scattered punctate bright foci corresponding to tiny calcifications. These findings in the appropriate clinical context should raise the possibility of Em. A corresponding axial unenhanced CT image (B) shows that the three lesions demonstrate curvilinear peripheral calcifications (*), again, evocative of the diagnosis. (C) An axial T2-weighted fat-suppressed image shows three ovoid lesions in the right hepatic lobe. There are numerous tiny cysts $\left(^{*}\right)$ at the periphery of the lesions. (D) An axial post-gadolinium enhanced T1weighted fat-suppressed image shows that the lesions have irregular margins and are predominantly poorly enhancing. These features are typical for $E m$. This figure appears in color at www.ajtmh.org.

In eight patients, investigation for abdominal symptoms led to the initial finding of a hepatic lesion. In eight patients, including two patient (patients 4 and 11) with known malignancies which prompted the screening, the initial finding of a liver lesion was incidental, following imaging for an unrelated indication. In most cases, malignancy was initially suspected as the explanation for the liver lesion(s). Hepatic lesions ranged in size from 0.7 to $14.1 \mathrm{~cm}$ (Figure 2).

Seven patients had an elevated alanine transaminase enzyme level between two and 13 times the upper limit of normal at the time of diagnosis; four patients had mild cholestatic enzyme elevations. No patient had an elevated eosinophil count.

Patients 1 and 8 required three liver biopsies before the diagnosis was made; three others (patients 2, 6, and 10) required two liver biopsies to establish the diagnosis. In six patient (patients $3,7,9,11,15$, and 17), the initial diagnostic specimen was obtained only at surgical excision Figure 3.

Albendazole therapy was initiated in all patients as soon as possible after diagnosis (Table 1). One patient declined to continue it post-surgery, and one had an elevation of her transaminase enzymes to 20 times the upper limit of normal, replicated on rechallenge, but is tolerating mebendazole (although the recommended dose requires 36, $100 \mathrm{mg}$ tablets daily).

In total, nine patients have undergone surgery. In the six in whom the diagnosis was made only after surgery, primary (patients 3, 7, 15, and 17), or metastatic (patient 11) tumor was suspected. In one patient (patient 9), it was an unexpected finding at liver explant in the course of transplantation for hepatitis $\mathrm{C}$ and alcohol-related cirrhosis. Only three patients (patients 4, 5, and 8) underwent planned surgery with curative intent following a biopsy diagnosis; one patient (patient 5) then declined albendazole, one patient (patient 4) has completed 2 years of albendazole with presumptive cure and has remained disease free at follow-up, and one patient (patient 8) was found to have extrahepatic extension of the disease at operation. The patient who underwent complete excision during liver transplantation has also completed 2 years of therapy for presumed cure.

In terms of complications, one patient (patient 13) has experienced secondary bacterial infection in a necrotic parasitic liver mass, and several patients have experienced significant persistent pain at the site of disease and/or surgery. None of our patients have had clinical or imaging evidence of distant metastases. However, four patients (patients 3, 7, 8, and 15) had surgical and/or histological findings of peritoneal or pleural involvement. One patient (patient 17) died of postoperative complications following excision of what was thought preoperatively, to be cholangiocarcinoma, after the incidental finding of a hepatic lesion on imaging for an unrelated problem.

\section{DISCUSSION}

The unprecedented appearance of 17 human cases of $A E$ in a circumscribed region of continental North America over 7 

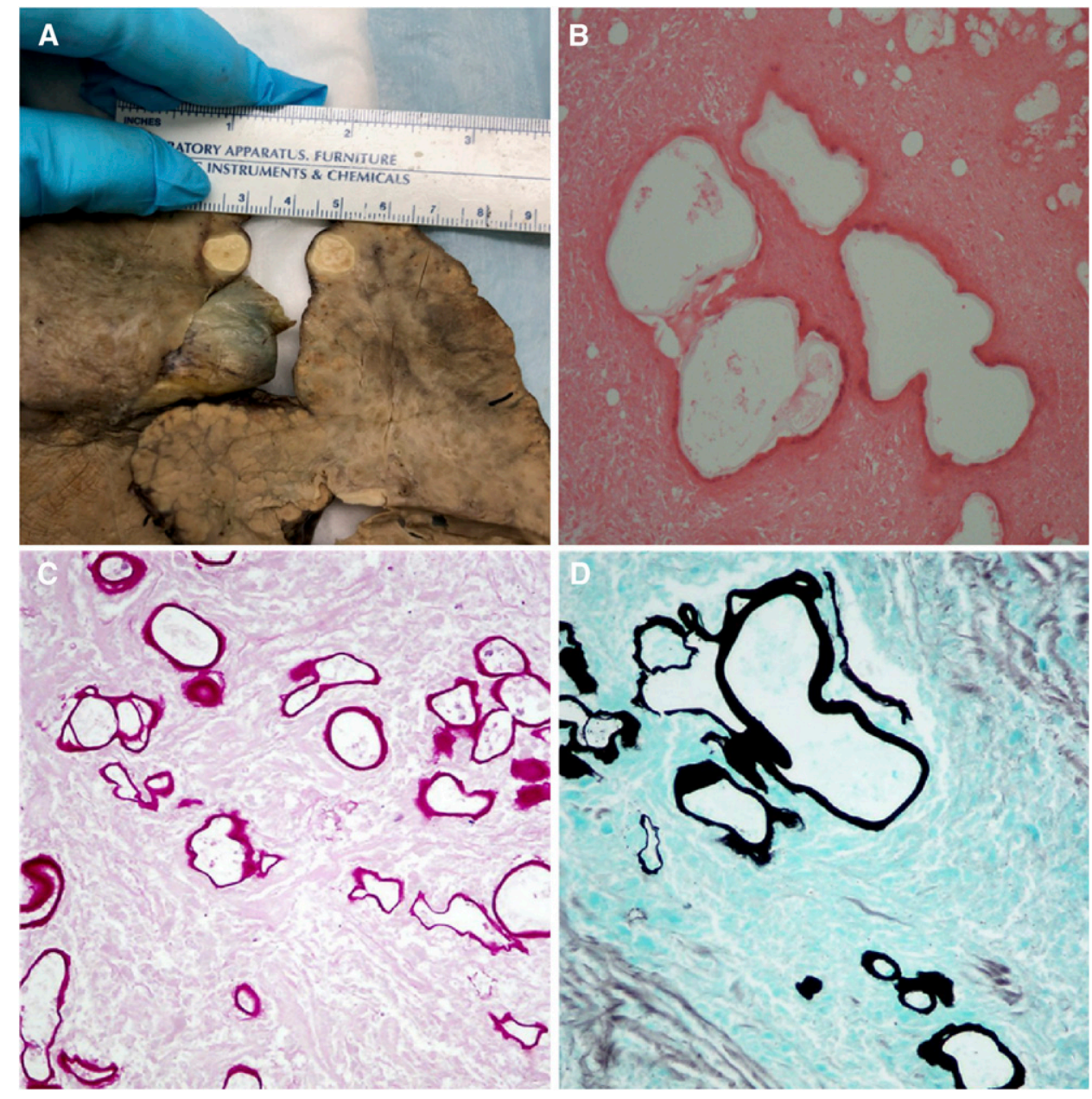

FigURE 3. Alveolar echinococcosis in explanted liver (before transplant). (A) Patient 9: histology preparations-routine H\&E staining (B) shows microcystic structures lined by a laminated grayish membrane on a background of necrosis. Staining with GMS and PAS (C and D) further highlights the laminated membranes. This figure appears in color at www.ajtmh.org.

years is a novel and significant development in the epidemiology of this disease. The annual incidence of diagnosed AE in Alberta of 0.064 per 100,000 inhabitants over the past 7 years is within the range observed in endemic countries of Europe-from 0.03 to $0.20 / 100,000$ inhabitants/year. ${ }^{10}$

Travel-related AE in North America has never been well documented despite millions of travelers to endemic areas of Europe over many decades; hence, the geographic and temporal clustering of our cases is compelling evidence of local transmission. In nine patients, local acquisition was confirmed definitively by the absence of any lifetime history of travel to an endemic area. Even in those who had ever been to Europe, their limited exposure history there was unlikely to explain their infection. This cluster of 17 human cases is also temporally and geographically associated with the emergence of a European-like strain of Em in local wildlife. Finally, in several cases, it has been possible to demonstrate the presence of an SNP unique to Canada (19).

The postulated greater virulence of the European strain may explain its emergence in humans and rapid dominance in animal hosts in North America. The observed concurrent reduction in the detection of the previously endemic North American sub-strain N2 in the animal reservoir following introduction of this more virulent strain is consistent with the competitive exclusion principle. ${ }^{34}$ The presence of Europeanlike strains of $E m$ in North America in wildlife, dogs, and humans since $2012^{25,27}$ is best explained by the recent importation of dogs or foxes from Europe because screening or anthelmintic treatment of imported animals is not required. ${ }^{25,35}$

A relatively high prevalence (from $25 \%$ to $60 \%$ ) of $E m$ has recently been found in urban coyotes in Calgary and Edmonton, Alberta. ${ }^{36-38}$ Five hundred to 1,000 coyotes are estimated to live within the city of Edmonton with similar numbers estimated for Calgary. The potential access of domestic dogs to infected rodents increases the risk of intestinal infection in pets. ${ }^{39}$ The presence of infected coyotes also increases the potential for contaminating garden produce and for the adherence of Em eggs to dogs' fur associated with "scent rolling" behavior involving coyote feces. Even at a low prevalence of infection, the presence of more than 125,000 domestic dogs in Calgary could significantly contribute to the parasite sylvatic cycle as well as serve as a potential direct source of human exposure.

Fifty (9.8\%) AE cases in a European registry between 1982 and 2009 had one or more immunosuppressive conditions before or at the time of diagnosis of $A E$, including five patients with organ transplants. ${ }^{9} \mathrm{An}$ accelerated disease course has 
been seen in AE patients with HIV coinfection. ${ }^{40,41}$ Six of our $17 \mathrm{AE}$ patients had received immunosuppressive therapies, a higher proportion than has been seen in Europe. These patients not only comprise a group at increased risk of $A E$ but may serve as a sentinel population for the disease in North America. The high proportion of immunocompromised individuals among our patient group suggests that we may be at an early stage of emergence of the disease in North America because these patients are likely to have shorter incubation periods. This conjecture is supported by the high proportion who were found incidentally, at an early stage before progression to symptomatic disease.

Imaging findings in AE are often initially interpreted as malignant, ${ }^{11}$ particularly in patients with a history of malignancy. The diagnosis of $A E$ would have been missed in patient 11 had the decision to excise the presumed malignant lesion not been made.

A biopsy may readily miss the diagnosis, as was the case with the initial biopsy in five of our patients, ${ }^{42}$ particularly if histopathologists lack familiarity with the disease. Serology and PCR may be helpful if histology is nondiagnostic. A biopsy diagnosis can critically inform the treatment strategy, specifically in regard to a decision around surgery. A team approach to care of $A E$ is desirable as decisions regarding the feasibility of curative surgery require surgical and imaging expertise. Pharmacologic treatment options remain extremely limited ${ }^{13}$ and are uniquely complicated in North America; the drug of choice, that is, albendazole, is very expensive in the United States and accessible only through a complex application process in Canada, although widely and cheaply available worldwide.

\section{CONCLUSION}

As a result of these new findings, clinicians in North America must now consider $A E$ in the differential diagnosis of mass lesions in the liver.

The emergence of SARS-CoV-2 is a reminder of the potential impact of emerging infections of zoonotic origin and of the importance of a One Health perspective. Animal studies to better understand and track local parasite epidemiology along with monitoring of the disease in human populations in North America are needed to inform public health policy.

Received December 11, 2020. Accepted for publication January 26, 2021.

Published online March 22, 2021.

Acknowledgments: We thank Sandra Cockfield who pressed for a repeat biopsy to diagnose patient 1 . We also thank Banu Sis who first made the pathological diagnosis and Jamil Kanji who cares for patient \# 16.

Disclosure: The University of Alberta (REB Pro00097735) approved the study.

Authors' addresses: Stan Houston, Department of Medicine/Public Health, University of Alberta, Edmonton, Canada, E-mail: shouston@ ualberta.ca. Sara Belga, Department of Medicine, Faculty of Medicine and Dentistry, University of Alberta, Edmonton, Canada, and Department of Medicine, Faculty of Medicine, University of British Columbia, Vancouver, Canada, E-mail: sara.belga@ubc.ca. Klaus Buttenschoen and Jutta Preiksaitis, Department of Surgery, Faculty of Medicine and Dentistry, University of Alberta, Edmonton, Canada, E-mails: klaus.buttenschoen@ualberta.ca and jutta@ualberta.ca. Ryan Cooper, Department of Medicine, Faculty of Medicine and Dentistry, University of Alberta, Edmonton, Canada, E-mail: rdcooper@ualberta.ca. Safwat Girgis, Department of Anatomical Pathology, University of Alberta, Edmonton, Canada, E-mail: sgirgis@ ualberta.ca. Bruno Gottstein, Institute of Parasitology, University of Bern, Bern, Switzerland, E-mail: bruno.gottstein@ifik.unibe.ch. Gavin Low and Philippe Sarlieve, Department of Radiology and Diagnostic Imaging, University of Alberta Faculty of Medicine and Dentistry, Edmonton, Canada, E-mails: low1@ualberta.ca and sarlieve@ ualberta.ca. Alessandro Massolo, Department of Biology, University of Pisa, Pisa, Italy, E-mail: alessandro.massolo@unipi.it. Clayton MacDonald, University of Alberta Faculty of Medicine and Dentistry, Alberta, Edmonton, Canada, E-mail: clayton.macdonald2@vch.ca. Norbert Müller, Department of Parasitology, Vetsuisse Faculty Bern, Bern, Switzerland, E-mail: norbert.mueller@vetsuisse.unibe.ch. Stephen Vaughan, Department of Infectious Diseases, Universiity of Calgary, Calgary, Canada, E-mail: stephen.vaughan@albertahealthservices.ca. Kinga Kowalewska-Grochowska, Provincial Laboratory of Public Health, University of Alberta Faculty of Medicine and Dentistry, Edmonton, Canada, E-mail: kinga.kowalewska-grochowska@albertaprecisionlabs.ca.

\section{REFERENCES}

1. Boireau P; Food and Agriculture Organization of the United Nations, World Health Organization, 2014. Multicriteria-Based Ranking for Risk Management of Food-Borne Parasites: Report of a Joint FAO/WHO Expert Meeting, September 3-7, 2012, FAO Headquarters, Rome, Italy. Rome, Italy, Geneva, Switzerland: Food and Agriculture Organization of the United Nations; World Health Organization.

2. Wen H, Vuitton L, Tuxun T, Li J, Vuitton DA, Zhang W, McManus DP, 2019. Echinococcosis: advances in the 21 st century. Clin Microbiol Rev 32: e0075-18.

3. Torgerson PR, Keller K, Magnotta M, Ragland N, 2010. The global burden of alveolar echinococcosis. PLoS Negl Trop Dis 4: e722.

4. Eckert J, Thompson RC, 2017. Historical aspects of echinococcosis. Adv Parasitology 95: 1-64.

5. Thompson RCA, 2017. Chapter two - biology and systematics of Echinococcus. Thompson RCA, Deplazes P, Lymbery AJ, eds. Advances in Parasitology, Vol. 95. London, UK: Academic Press, 65-109.

6. Corsini M, Geissbuhler U, Howard J, Gottstein B, Spreng D, Frey CF, 2015. Clinical presentation, diagnosis, therapy and outcome of alveolar echinococcosis in dogs. Vet Rec 177: 569.

7. Craig PS, Hegglin D, Lightowlers MW, Torgerson PR, Wang Q, 2017. Chapter two - echinococcosis: control and prevention. Thompson RCA, Deplazes P, Lymbery AJ, eds. Advances in Parasitology, Vol. 96. Academic Press, 55-158.

8. Eckert J, Deplazes P, 2004. Biological, epidemiological, and clinical aspects of echinococcosis, a zoonosis of increasing concern. Clin Microbiol Rev 17: 107-135.

9. Chauchet A et al., 2014. Increased incidence and characteristics of alveolar echinococcosis in patients with immunosuppressionassociated conditions. Clin Infect Dis 59: 1095-1104.

10. Vuitton DA, Demonmerot F, Knapp J, Knapp J, Richou C, Grenouillet F, Chauchet A, Vuitton L, Bresson-Hadni S, Millon L, 2015. Clinical epidemiology of human AE in Europe. Vet Parasitol 213: 110-120.

11. Brunetti E, Kern P, Vuitton DA, 2010. Expert consensus for the diagnosis and treatment of cystic and alveolar echinococcosis in humans. Acta Trop 114: 1-16.

12. Buttenschoen K, Gruener B, Carli Buttenschoen D, Reuter S, Henne-Bruns D, Kern P, 2009. Palliative operation for the treatment of alveolar echinococcosis. Langenbecks Arch Surg 394: 199-204.

13. Siles-Lucas M, Casulli A, Cirilli R, Carmena D, 2018. Progress in the pharmacological treatment of human cystic and alveolar echinococcosis: compounds and therapeutic targets. PLOS Negl Trop Dis 12: e0006422.

14. Aliakbarian M, Tohidinezhad F, Eslami S, Akhavan-Rezayat K, 2018. Liver transplantation for hepatic alveolar echinococcosis: literature review and three new cases. Infect Dis (Lond) 50: 452-459.

15. Spotin A, Boufana B, Ahmadpour E, Casulli A, Mahami-Oskouei M, Rouhani S, Javadi-Mamaghani A, Shahrivar F, Khoshakhlagh P, 2018. Assessment of the global pattern of genetic diversity in 
Echinococcus multilocularis inferred by mitochondrial DNA sequences. Vet Parasitol 262: 30-41.

16. Nakao M, Xiao N, Okamoto M, Yanagida T, Sako Y, Ito A, 2009. Geographic pattern of genetic variation in the fox tapeworm Echinococcus multilocularis. Parasitol Int 58: 384-389.

17. James E, Boyd W, 1937. Echinococcus alveolaris: (with the report of a case). Can Med Assoc J 36: 354-356.

18. Klein C, Massolo A, 2015. Demonstration that a case of human alveolar echinococcosis in Minnesota in 1977 was caused by the N2 strain. Am J Trop Med Hyg 92: 477-478.

19. Gamble WG, Segal M, Schantz PM, Rausch RL, 1979. Alveolar hydatid disease in Minnesota. First human case acquired in the contiguous United States. JAMA 241: 904-907.

20. Wilson JF, Rausch RL, 1980. Alveolar hydatid disease. A review of clinical features of 33 indigenous cases of Echinococcus multilocularis infection in Alaskan Eskimos. Am J Trop Med Hyg 29: 1340-1355.

21. Polish LB, Pritt B, Barth TFE, Gottstein B, O'Connell EM, Gibson PC, 2020. Echinococcus multilocularis: first European haplotype identified in the United States: an emerging disease? Clin Infect Dis. 2020 Mar 21 (Epub ahead of print): ciaa245. https:// doi.org/10.1093/cid/ciaa245.

22. Bartel MH, Seesee FM, Worley DE, 1992. Comparison of Montana and Alaska isolates of Echinococcus multilocularis in gerbils with observations on the cyst growth, hook characteristics, and host response. J Parasitol 78: 529-532.

23. Hildreth MB, Sriram S, Gottstein B, Wilson M, Schantz PM, 2000. Failure to identify alveolar echinococcosis in trappers from South Dakota in spite of high prevalence of Echinococcus multilocularis in wild canids. J Parasitol 86: 75-77.

24. Massolo A et al., 2019. European Echinococcus multilocularis identified in patients in Canada. New Engl J Med 381: 384-385.

25. Gesy K, Hill JE, Schwantje H, Liccioli S, Jenkins EJ, 2013. Establishment of a European-type strain of Echinococcus multilocularis in Canadian wildlife. Parasitology 140: 1133-1137.

26. Gesy KM, Jenkins EJ, 2015. Introduced and native haplotypes of Echinococcus multilocularis in wildlife in saskatchewan, Canada. J Wildl Dis 51: 743-748.

27. Jenkins EJ, Peregrine AS, Hill JE, Somers C, Gesy K, Barnes B, Gottstein B, Polley L, 2012. Detection of European strain of Echinococcus multilocularis in North America. Emerg Infect Dis 18: $1010-1012$.

28. Jones KE, Patel NG, Levy MA, Storeygard A, Balk D, Gittleman JL, Daszak P, 2008. Global trends in emerging infectious diseases. Nature 451: 990-993.

29. Gottstein B, Schantz PM, Todorov T, Saimot AG, Jacquier P, 1986. An international study on the serological differential diagnosis of human cystic and alveolar echinococcosis. Bull World Health Organ 64: 101-105.

30. Xiao $\mathrm{N}$ et al., 2003. Evaluation of use of recombinant Em18 and affinity-purified Em18 for serological differentiation of alveolar echinococcosis from cystic echinococcosis and other parasitic infections. J Clin Microbiol 41: 3351-3353.

31. Muller N, Frei E, Nunez S, Gottstein B, 2007. Improved serodiagnosis of alveolar echinococcosis of humans using an in vitroproduced Echinococcus multilocularis antigen. Parasitology 134: 879-888.

32. Muller N, Zimmermann V, Forster U, Bienz M, Gottstein B, Welle $M, 2003$. PCR-based detection of canine Leishmania infections in formalin-fixed and paraffin-embedded skin biopsies: elaboration of a protocol for quality assessment of the diagnostic amplification reaction. Vet Parasitol 114: 223-229.

33. Trachsel D, Deplazes $P$, Mathis A, 2007. Identification of taeniid eggs in the faeces from carnivores based on multiplex PCR using targets in mitochondrial DNA. Parasitology 134:911-920.

34. Bremermann HJ, Thieme HR, 1989. A competitive exclusion principle for pathogen virulence. J Math Biol 27: 179-190.

35. Massolo A, Liccioli S, Budke C, Klein C, 2014. Echinococcus multilocularis in North America: the great unknown. Parasite 21: 73.

36. Luong LT, Chambers JL, Moizis A, Stock TM, St Clair CC, 2018. Helminth parasites and zoonotic risk associated with urban coyotes (Canis latrans) in Alberta, Canada. J Helminthol 94: e25.

37. Liccioli S, Kutz SJ, Ruckstuhl KE, Massolo A, 2014. Spatial heterogeneity and temporal variations in Echinococcus multilocularis infections in wild hosts in a North American urban setting. Int J Parasitol 44: 457-465.

38. Catalano S, Lejeune M, Liccioli S, Verocai GG, Gesy KM, Jenkins EJ, Kutz SJ, Fuentealba C, Duignan PJ, Massolo A, 2012. Echinococcus multilocularis in urban coyotes, Alberta, Canada. Emerg Infect Dis 18: 1625-1628.

39. Nation PN, St Clair CC, 2019. A forensic pathology investigation of dismembered domestic cats: coyotes or cults? Vet Pathol 56: 444-451.

40. Sailer M, Soelder B, Allerberger F, Zaknun D, Feichtinger $H$, Gottstein B, 1997. Alveolar echinococcosis of the liver in a sixyear-old girl with acquired immunodeficiency syndrome. $J$ Pediatr 130: 320-323.

41. Zingg $W$ et al., 2004. Alveolar echinococcosis of the liver in an adult with human immunodeficiency virus type-1 infection. Infection 32: 299-302.

42. Stojkovic M, Mickan C, Weber TF, Junghanss T, 2015. Pitfalls in diagnosis and treatment of alveolar echinococcosis: a sentinel case series. BMJ Open Gastroenterol 2: e000036. 